\title{
CUERPO, EMOCIONES Y LUGAR: APROXIMACIONES TEÓRICAS Y METODOLÓGICAS DESDE LA GEOGRAFÍA
}

\author{
Anna Ortiz Guitart \\ Dpto. de Geografía, Universitat Autònoma de Barcelona, \\ 08193 Bellaterra, Cerdanyola del Vallès \\ anna.ortiz@uab.es
}

\begin{abstract}
Resumen: Este artículo presenta las principales aportaciones sobre los cuerpos en geografía. Se presenta una amplia variedad de artículos publicados en revistas de geografía anglosajonas ya que, sin lugar a dudas y de manera más notable, ha contribuido a la reflexión sobre este campo del saber. La mayoría de estos artículos están publicados en revistas cuya línea editorial se manifiesta particularmente sensible a las perspectivas sociales y culturales en geografía. En menor medida se presentan aportaciones procedentes de la geografía española y latinoamericana ya que su presencia ha sido y continúa siendo, especialmente en el caso español, casi inexistente.
\end{abstract}

Palabras clave: geografía social y cultural, feminismo, cuerpo, lugar, revisión teórica.

\begin{abstract}
Body, emotions and place: theoretical and methodological approaches in Geography. This article shows the main contributions made in geography on the body. It presents a wide variety of articles published in Anglo-American journals of geography that most notably have contributed to the debate on this field of knowledge. Most of these articles are published in journals whose editorial appears particularly sensitive to social and cultural perspectives in geography. To a lesser extent it presents contributions from Spanish and Latin American geography as their presence has been and continues to be, especially in the Spanish context, almost nonexistent.
\end{abstract}

Keywords: social and cultural geography, feminism, body, place, theoretical review. 


\section{Introducción}

Este artículo presenta las principales aportaciones teóricas y metodológicas realizadas en geografía sobre el cuerpo, las emociones y el lugar. La mayoría de estas contribuciones proceden de la geografía anglosajona, y más concretamente de la geografía feminista, ya que ha sido en este ámbito donde, sin lugar a dudas y de manera más notable, se ha contribuido más intensamente a la reflexión sobre este campo del saber. En menor medida se presentan aportaciones procedentes de la geografía española y latinoamericana ya que su presencia ha sido y continúa siendo, especialmente en el caso español, casi inexistente.

A lo largo del artículo se hace evidente como las investigaciones sobre cuerpo, emociones y lugar son muy fructíferas y relevantes en la geografía anglosajona desde los años noventa del siglo XX, y extremadamente escasas en cuanto al volumen (pero no en cuanto a calidad) en la geografía española y latinoamericana. Algunas de las razones que podrían explicar el escaso interés hacia esta perspectiva y la residualidad de sus publicaciones podrían ser las siguientes. En primer lugar, las perspectivas sociales y culturales en la geografía española han sido muy limitadas comparadas con la geografía anglosajona, francesa o brasileña, destacándose la publicación de algunos estudios sobre género y ciudad (García Ramón et al., 2003). Bastaría fijarse en los nuevos planes de estudio de Grado en Geografía y Ordenación del Territorio de las distintas universidades españolas para constatar el auge que en los últimos años gozan materias relacionadas con la Ordenación del Territorio, el medio ambiente y los Sistemas de Información Geográfica y la marginalidad de las perspectivas más sociales y culturales. En segundo lugar, las geografías de las emociones, tan desarrolladas desde principios del siglo XXI en el mundo anglosajón (con libros como el de Davidson et al., 2007 y numerosos artículos publicados en las principales revistas de geografía, todas ellas indexadas) han visibilizado notablemente la corporalidad de las emociones, siendo este mismo enfoque totalmente inexistente en las revistas de geografía españolas. Finalmente, el interés por la identidad, la interseccionalidad o la edad brillan por su ausencia en los estudios geográficos españoles, salvo en contadas excepciones (Prats et al., 2012). Este desinterés puede explicar también por qué el 'cuerpo', tan interrelacionado con estos ejes de análisis, sigue 'escondido' en la geografía española.

La estructura del artículo es la siguiente. En primer lugar, se hace una breve presentación de la corporalidad en geografía. En segundo lugar, se presenta una amplia variedad de artículos publicados en revistas de geografía anglosajonas. La mayoría de estos artículos están publicados en revistas cuya línea editorial se manifiesta particularmente sensible a las perspectivas sociales y culturales en geografía. Estas son: Social and Cultural Geography, Gender, Place and Culture, y Geoforum. En tercer lugar, se presentan los escasos trabajos, pero no por pocos menos interesantes, procedentes de la geografía española y latinoamericana. Posteriormente, se exponen las principa- 
les apuestas metodológicas que tienen como base el cuerpo como herramienta de investigación. Ya por último, en las reflexiones finales se apuntan algunas de las posibles razones de la invisibilidad del 'cuerpo' en la geografía española. Los autores/as citados aportan interesantes y novedosas perspectivas de análisis sobre el estudio del cuerpo en geografía que abarcan temáticas variadas y escalas de análisis amplias que pueden aportar ideas para futuras líneas de investigación sobre el tema.

Sin ninguna pretensión de exhaustividad, este artículo recopila algunos trabajos que bajo distintos ejes (la fluidez de los cuerpos, cuerpos vestidos y desnudos, cuerpos saludables y cuerpos enfermos, cuerpos jóvenes y móviles) presentan la interrelación entre cuerpo, emociones y lugar. El objetivo de este artículo es, de esta forma, dar a conocer estos trabajos con el fin de mostrar otras formas de 'hacer' y 'entender' la geografía.

\section{Cuerpos y geografía}

"Un cuerpo, aunque no todos los estudiosos de la geografía lo crean, es un lugar. Se trata del espacio en el que se localiza el individuo, y sus límites resultan más o menos impermeables respecto a los restantes cuerpos. Aunque no cabe duda de que los cuerpos son materiales y poseen ciertas características como la forma y el tamaño, de modo que, inevitablemente, ocupan un espacio físico, lo cierto es que su forma de presentarse antes los demás y de ser percibido por ellos varía según el lugar que ocupan en cada momento" (McDowell, 2000, p. 59). Con esta claridad expositiva empieza el capítulo de Linda McDowell dedicado al estudio del cuerpo en geografía en su libro Género, identidad y lugar (2000), de lectura obligada para entender la importancia de incorporar la perspectiva de género en geografía.

El cuerpo es lo que somos, a través de él experimentamos nuestras emociones y nos conectamos con el mundo. Los cuerpos ocupan espacios y, a la vez, son espacios en sí mismos; son lugares físicos donde las relaciones de género, clase y etnia se encuentran y son practicadas. Los cuerpos pueden ser mapas de deseo, disgusto, placer, dolor, odio y amor y son, además, los primeros objetos de inscripción (superficies donde los valores, la moralidad y las leyes sociales se inscriben) (Teather, 1999). El lenguaje corporal, la masa corporal, la forma de vestir o las marcas hechas sobre el cuerpo son prácticas concretas que actúan en la construcción de las identidades, haciendo que éstas puedan ser leídas o reinterpretadas de formas muy diferentes (Hopkins, 2010).

Entender la experiencia del cuerpo, tanto la de la persona que realiza la investigación como la de las personas investigadas, puede ser esencial para entender las relaciones de las personas con los ambientes físicos y sociales (Longhurst, 1997; Butler, 1999). Los cuerpos juegan un papel esencial a la hora de configurar las experiencias de las personas en los lugares. Y la práctica de nuestros cuerpos (con su género, sus 
preferencias sexuales, sus habilidades físicas, su edad, su color o su etnicidad) es única y depende de los contextos específicos espaciales, temporales y culturales donde se sitúen. De ahí que los cuerpos no pueden ser entendidos como proyectos individuales o entidades autónomas sino más bien como productos relacionalmente constituidos (Landzelius, Michael, 2005; Bethan, 2006; Abrahamsson y Simpson, 2011).

Tradicionalmente la geografía no ha considerado cuestiones relacionadas con el cuerpo "ya que la tradición sitúa a la disciplina en el terreno público, con total exclusión de lo privado, y el cuerpo, con sus atributos, su conducta y su sexualidad siempre se ha tenido por un interés estrictamente privado, aunque (...) los estudios feministas más recientes han demostrado que también el cuerpo es una construcción de los discursos y las actuaciones públicas que se producen a distintas escalas" (McDowell, 2000: 61). Más allá de esta reflexión Longhurst et al. (2008) consideran que las emociones y los sentimientos pertenecen a la categoría de la vida cotidiana y por esta razón se han dejado al margen de la investigación; demasiado prosaicos y banales para tenerse en cuenta. Partiendo del conocimiento situado, estas investigadoras se posicionan "no como autónomas o académicas racionales sino como personas que a veces sienten emociones irracionales", animando a "los geógrafos empezar a hablar desde un lugar corporizado, más que desde un lugar elevado" (Longhurst et al., 2008: 213).

Longhurst (2001) hace un repaso por la historia reciente del pensamiento geográfico y señala que, si bien es cierto que la geografía humanística visibilizó los cuerpos en geografía al enfatizar la subjetividad de los geógrafos/as y del fenómeno observado con el fin de significar el lugar, esta corriente olvidó reconocer la especificidad de los cuerpos (con sus diferentes colores, formas, habilidades, características, sexos, género...). No fue hasta la llegada del postmodernismo en geografía en los años noventa y la influencia que ejercieron teóricos postmodernos como Judith Butler, Gilles Deleuze y Michel Foucault (y sus aportaciones en la reconceptualización de la "construcción" del cuerpo como objeto generizado, como efecto de las relaciones de poder, etc.), que temas como las representaciones, las identidades y los cuerpos ocuparon un lugar en geografía (Longhurst, 2001).

Quisiera terminar este primer apartado señalando los numerosos libros centrados en la corporalidad en geografía publicados por editoriales anglosajonas en la segunda mitad de los años noventa: Sensuous geographies: body, sense and place de Paul Rodaway (1994), The body and the city: psychoanalysis, space and subjectivity de Steve Pile (1996), Body space: destabilising geographies of gender and sexuality de Nancy Duncan (1996), Mapping desire: geographies of sexualities (1995) y Consuming geographies: we are where we eat de David Bell y Gill Valentine (1997), Places through the body de Heidi Nast y Steve Pile (1998), New frontiers of space, bodies and gender de Rosa Ainley (1998), Embodied Geographies de Elizabeth Kenworthy Teather (1999) y Mind and body spaces: geographies of illness, impairment and disability de Hester Parr y Ruth Butler (1999). 


\section{Los cuerpos en la geografía anglosajona}

Con el fin de presentar una panorámica general de las investigaciones realizadas en el mundo anglosajón sobre el cuerpo, las emociones y el lugar desde una perspectiva geográfica, se considera pertinente clasificar el gran volumen de trabajos encontrados en cuatro ejes temáticos: 1) la fluidez de los cuerpos, a partir de los trabajos de la geógrafa Robyn Longhurst, 2) los cuerpos vestidos y desnudos, a partir de las repercusiones de la moda y la cultura en las formas de vestir de los hombres y mujeres, 3) los cuerpos saludables y cuerpos enfermos, a partir de las geografías del cuidado centradas en las espacialidades del cuerpo y, finalmente, 4) los cuerpos jóvenes y móviles, a partir de las experiencias y las prácticas de las personas jóvenes en su vida y espacios cotidianos.

\subsection{La fluidez de los cuerpos: las aportaciones de la geógrafa neozelandesa Robyn Longhurst}

Las razones por las cuales los cuerpos empezaron a ser tan populares en los años noventa del siglo XX en ciencias sociales y humanidades se debieron a que la atención sobre ellos permitió criticar la universalidad y la objetividad del conocimiento. El cuerpo se convirtió en el lugar por excelencia para explorar nuevas formas de entender el poder y las relaciones sociales entre personas y lugares. En este apartado nos centramos en el trabajo de la geógrafa neozelandesa Robyn Longhurst por ser una de las geógrafas feministas más influyentes y productivas en el estudio del cuerpo en geografía. Los estudios feministas fueron y han sido centrales en la nueva forma de pensar el cuerpo y teorizarlo. Desde principios de los años noventa, las investigaciones geográficas feministas han querido mostrar que el cuerpo nunca es una entidad fija y acabada, sino plástica y maleable. Longhurst (2001) considera que el poco interés por el cuerpo (con sus desórdenes y sus partes oscuras y sucias) en geografía se debe a que, tradicionalmente, las políticas de producción científica han sido dominadas por las necesidades e intereses de los hombres.

Otra idea interesante señalada por Longhurst (1997) es que los cuerpos han sido construidos por la geografía como parte de la Otredad (es decir, como categoría inferior a la mente). Mientras que algunas nociones positivas como la razón, el sujeto, la consciencia, la actividad y la masculinidad se relacionan con la mente, otras negativas como la pasión, el objeto, el inconsciente, la pasividad y la feminidad se relacionan con el cuerpo.

Especialmente relevantes son sus investigaciones sobre los cuerpos de las mujeres embarazadas. Según Longhurst (1999, 2001), las mujeres embarazadas transgreden y distorsionan la realidad y el orden al no respetar las fronteras, las posiciones y las reglas. La mujer embarazada chilla (se las construye como personas con una sensibilidad a flor de piel), vomita (con sus náuseas matinales), orina frecuentemente, rompe aguas, etc. 
Es, por tanto, un cuerpo abyecto, peligroso e inestable; un cuerpo que necesita ser controlado porque los fluidos provocan sentimientos de deshonra, bajeza y humillación. Mientras que los cuerpos de los hombres (especialmente si son heterosexuales, blancos y buen capacitados físicamente) tienden a construirse como sólidos, fuertes y con unas fronteras corporales seguras y bajo control, los cuerpos de las mujeres embarazadas se construyen como vulnerables. Para su investigación la geógrafa entrevistó a un grupo de mujeres embarazadas y analizó su relación con los espacios públicos de Hamilton (Nueza Zelanda). Sus resultados muestran como estas mujeres se sienten incómodas en ciertos espacios públicos (llenos de humo, con pocos lavabos, con asientos incómodos) y cambian sus hábitos quedándose más en casa, haciendo menos deporte, no saliendo por la noche y dejando de trabajar de forma remunerada.

Ciertamente, a menudo se critica que los cuerpos que más llaman la atención para estudiar son aquellos construidos como los Otros (las mujeres embarazadas, las mujeres lesbianas, los hombres homosexuales, los 'negros', los pobres, las personas mayores, las personas discapacitadas, etc.) y que, contrariamente, los cuerpos de los hombres heterosexuales, adultos, 'blancos' y con todas las capacidades físicas están totalmente relegados. Pero Longhurst (2001) estudia precisamente los cuerpos de estos hombres y su relación con los lavabos. Considera que los lavabos son espacios donde los cuerpos se re(hacen) y se preparan para el escrutinio público. A partir del análisis de las entrevistas realizadas a un grupo de hombres neozelandeses muestra distintos resultados: el discurso de los fluidos y de los flujos está totalmente ausente de sus narrativas, los lavabos son descritos como espacios funcionales y no como espacios de relajación, los hombres que comparten lavabos con otros hombres temen contagiarse con fluidos de otros cuerpos y los hombres sienten que tienen un cuerpo sólido y fuerte cuando en realidad, como todos los cuerpos, no dejan de ser viscosos y líquidos. La incorporación de los lavabos como lugares de análisis es otra de las aportaciones de la geógrafa ya que, según la autora, ignorar los espacios cerrados es olvidar lo íntimo, la femenino, lo queer, lo banal y la Otredad.

\subsection{Cuerpos vestidos y desnudos: entre la subjetividad y la espacialidad}

Continuando con el trabajo de Longhurst (2005), los vestidos y las modas son construcciones culturales de las subjetividades corporales que están íntimamente ligadas a las espacialidades. Esta autora muestra como las mujeres profesionales embarazadas que continúan trabajando durante el embarazo deciden vestir "normal" (con faldas ajustadas o tacones) como estrategia para que no se las juzgue como irracionales, emocionales o poco profesionales. Se espera de ellas que se adapten a la sociedad del consumo, vayan a la moda y sean atractivas; de ahí que su presencia se mantenga en los lugares de trabajo, los restaurantes y los centros deportivos. Si tenemos en cuenta que somos la mayor parte del tiempo 'cuerpos vestidos', como asegura la autora, no es nin- 
guna frivolidad decir que la moda es un elemento clave en la construcción de nuestra identidad y que, sin lugar a dudas, la clase social, el género, la sexualidad y la etnicidad se expresan a través de la producción, el consumo y las formas de vestir.

En la misma línea, Longhurst (2001) examina, a través de los cuerpos de los hombres y mujeres profesionales que trabajan en empresas privadas, la conducta del cuerpo y la presentación de éste como "profesional" y respetable. Nuevamente aparece la moda y la ropa (el traje en los hombres y el traje chaqueta en las mujeres) como esenciales para crear una ilusión del cuerpo como algo autónomo, controlado y racional. Otras investigaciones que relacionan cuerpo, moda y consumo (esta vez consumo alimentario) son Crewe (2001) y Mather y Mackenzie (2006).

Desde otra óptica, Colls (2004 y 2006) analiza las experiencias de las mujeres cuando van a comprar ropa (escoger la talla adecuada, probarse un vestido, encerrarse en un cambiador y compararse con otros cuerpos). La geógrafa analiza estas prácticas que acompañan al acto de comprar a través de grupos de discusión y entrevistas con mujeres y observa como el consumo de ropa está íntimamente relacionado con el conocimiento íntimo del cuerpo.

En otro contexto geográfico y cultural, en Estambul (Turquía), la investigación etnográfica de Gökariksel (2009) estudia la producción del cuerpo como espacio religioso y las experiencias espaciales y cotidianas de las mujeres que cubren o descubren su cabeza con el velo. A partir de una interesante reflexión sobre el hecho religioso, la autora muestra como el acto de vestirse puede ser la forma más visible y fácil a la hora de identificar la religiosidad o la secularidad de una persona.

\subsection{Cuerpos saludables y enfermos: la geografía de los cuidados}

Cuidarse (para estar en plena forma) y cuidar de las personas es una condición indispensable de la especie humana. Sin embargo, históricamente todo lo relacionado con el cuidado y las emociones han sido facetas desvalorizadas. Solamente cuando las mujeres, las principales provisoras del trabajo de atención al otro, se han incorporado significativamente al trabajo remunerado la provisión de cuidados ha empezado a considerarse en la agenda política. Numerosos estudios reflexionan sobre los cuidados corporales (a través del yoga, por ejemplo, con Lea, 2009), los cuidados interpersonales, la jerarquía de cuidados y su intersección con el género, la etnia y la clase (Dyer et al., 2008; Wainwright et al., 2010), así como los espacios dónde se dan los distintos cuidados (Atkinson et al., 2011). En la cultura occidental, señalan Dyer et al. (2008), donde priorizamos lo cerebral frente a lo emocional y lo físico, la autonomía frente a la dependencia y la disciplina frente a lo incontrolable e incontrolado, los trabajos corporales y de cuidados, donde el contacto íntimo (dar de comer, lavar, vestir) juega un papel esencial, se ven como tareas serviles y sucias. 
El cuidado del propio cuerpo es el eje principal del trabajo de McCormack (1999), Atkinson (2011) y Little (2012). El primer trabajo analiza cómo se construyen las imaginarios corporales (el 'cuerpo en buena forma' y los discursos entorno al riesgo, la flexibilidad y la responsabilidad). El segundo examina las geografías del cuidado centrándose en las espacialidades del cuerpo en relación a la práctica de la cirugía estética (una práctica vista por algunas personas como una tecnología al servicio del patriarcado opresivo o por otras como una práctica para mejorar la identidad y aliviar angustias de los seres humanos). Finalmente, el tercer trabajo reflexiona sobre el bienestar, las prácticas terapéuticas y el cuerpo, así como la relación entre feminidad y salud. El spa, el espacio terapéutico escogido para su investigación, resulta ser el lugar ideal para, a través de una ambientación lujosa y relajante, promover el bienestar y la salud del cuerpo. Sin embargo, como apunta la autora, el spa puede verse también como un espacio de vigilancia donde los cuerpos están constantemente sujetos a miradas disciplinarias.

Nuestra sociedad nos recuerda constantemente que cuidarse y mantenerse en forma es una de las premisas básicas para llevar una vida saludable. Eso sí, la publicidad de los gimnasios animan a hombres y a mujeres a mantenerse en forma con distintas actividades. Así, por ejemplo, a las mujeres se las anima para hacer aerobic, mientras que a los hombres se les anima para aumentar la musculatura. Las diferencias sexuales crean espacios femeninos y masculinos y estos lugares sexuados ayudan, a la vez, a crear cuerpos femeninos y masculinos. En torno a estos temas trata el artículo de Johnston (1996). La geógrafa estudia el cuerpo de las mujeres que practican culturismo en Hamilton (Nueva Zelanda) y muestra como su corporalidad transgresora proporciona un desafío a las construcciones estereotipadas de la feminidad y la masculinidad. Estas mujeres trabajan su cuerpo y aumentan sus músculos que, en sus cuerpos femeninos, se ven como peligrosos y 'fuera de lugar'. Deconstruye, de esta forma, las nociones hegemónicas de sexualidad, sexo y corporalidad al mostrar como los cuerpos de las culturistas y los gimnasios resultan lugares de cambio.

Los trabajos de Parr (2002a y 2002b) aportan un grado más de complejidad al tratar el cuidado del cuerpo a partir de su interrelación con el espacio, el conocimiento y el poder. Para el autor los discursos en los medios de comunicación, la medicina, la cultura del consumo y la industria de la moda determina nuestras necesidades materiales, placeres, posibilidades y limitaciones para producir geográficamente e históricamente normas específicas sobre cómo deben producirse nuestros cuerpos (en relación a la forma, el tamaño, el atractivo sexual, etc.) (Parr, 2002a). Señala, además, que no sólo el espacio real constituye e inventa los cuerpos orgánicamente sino que los cuerpos son constituidos nuevamente a través del espacio virtual (Parr, 2002b). A menudo se dice que el ciberespacio descorporaliza al cuerpo, que lo vuelve invisible e irrelevante en el mundo virtual; sin embargo, lo que si facilita, sin lugar a dudas, es la transformación de las identidades no asumidas (de género, de etnia...). Su investigación resulta de especial interés para la geografía porque trata una cuestión particularmente pertinente y novedosa: las fronteras en el espacio virtual. 
Pero no todos los trabajos giran entorno a los cuerpos saludables y en buena forma. Herrick (2007) y Colls (2007) analizan, desde el marco de rematerialización de la geografía social y cultural, la obesidad del cuerpo y la materialización de los cuerpos gordos. Porque en Occidente mientras que el cuerpo ideal es el cuerpo controlado y delgado, el cuerpo que está protegido contra la erupción y que tiene bajo control los procesos internos, el cuerpo insano, feo y 'fuera de lugar' está representado por el cuerpo obeso. Los cuerpos discapacitados (Sothern, 2007) y los cuerpos enfermos (Moss y Dick, 1996 y 1999; Malins et al., 2006) son analizados también por las geografías del cuerpo. El trabajo de Moss y Dick (1996 y 1999) es especialmente interesante para conocer el carácter fluido de la identidad de los sujetos y la identidad del cuerpo. Su investigación se centra en un grupo de mujeres diagnosticadas con enfermedades crónicas (esclerosis múltiple y síndrome de la fatiga crónica). Las geógrafas observan como a partir del diagnóstico de la enfermedad, la vida cotidiana de las mujeres enfermas se ve alterada, sus identidades fragmentadas y sus vidas abocadas hacia lo imprevisible, lo incierto y lo inestable. Las entrevistas realizadas a estas mujeres muestran hasta qué punto construyen identidades múltiples según el lugar. Así, por ejemplo, algunas mujeres actúan a veces de forma 'normal' en los lugares de trabajo por miedo a perderlos y lo hacen escondiendo el dolor, la fatiga y el malestar que les provoca su enfermedad. Otra investigación relacionada con los cuerpos enfermos es la de Malins et al. (2006), en la que se muestra la relación de los cuerpos de las mujeres drogadictas con los espacios públicos. A partir de entrevistas con mujeres que se inyectan drogas y de la posterior visita a los espacios públicos de Melbourne (Australia) en los que se inyectan, las investigadoras muestran cómo las características de estos espacios (forma, visibilidad, limpieza, luz, posición de las cámaras de seguridad, papeleras, etc.) son determinantes para mediar riesgos relacionados con la seguridad, la violencia o la infección.

\subsection{Cuerpos jóvenes y móviles: espacios de transgresión y de lucha}

A través de diferentes escalas geográficas (el cuerpo, el hogar y la comunidad, la nación y el mundo globalizado), Hopkins (2010) se acerca al conocimiento de las experiencias y las prácticas de las personas jóvenes en su vida cotidiana. Los jóvenes comunican sus identidades a través del cuerpo y éstas pueden ser negociadas (adoptadas, modificadas, resistidas o contestadas) de maneras distintas y actuadas a través de prácticas particulares como el lenguaje del cuerpo, la vestimenta u otras marcas corporales. Estudiar el cuerpo puede ayudar a entender mejor las relaciones entre edad, los ciclos de vida (Hopkins y Pain, 2007) e identidad, ya que el cuerpo nunca es fijo sino múltiple y producido en relación a otros cuerpos reales, recordados o imaginados.

En el editorial Colls y Hörschelmann (2009), del número monográfico de la revista Children's Geographies sobre geografías del cuerpo, se reflexiona sobre el hecho de que las experiencias espaciales del cuerpo de los niños/as y jóvenes han sido escasa- 
mente exploradas y consideradas. Uno de los artículos incluidos en este número es el de Ansell (2009) que analiza la vulnerabilidad de los cuerpos infantiles que padecen SIDA en Lesotho. Como consecuencia de la enfermedad (unida a la malnutrición y a las disfunciones emocionales), no ya sólo de los más pequeños sino de sus familiares, los cuerpos de los chicos y chicas experimentan cambios en su materialidad y corporeidad. La investigación muestra que la escuela juega un papel esencial para mitigar los impactos de la enfermedad y prevenir, a través del conocimiento del propio cuerpo, el contagio. En el mismo número se encuentra también el trabajo de Herrera et al. (2009) que analizan como los cuerpos de los jóvenes que viven en la calle en Puebla (México) expresan y experimentan sus vidas y sus identidades. El control que ejercen sobre sus cuerpos -como lugares de autodefinición, resistencia y transgresión-, así como, la creatividad y la libertad que este hecho genera, les permite crear una identidad social y un sentimiento de pertenencia necesario para su empoderamiento personal. Con resultados muy similares al trabajo anterior, Beazley (2003) observa que los espacios de vida cotidiana de los niños de la calle (estaciones de autobús, lavabos, intersecciones de tráfico, parques, mercados o espacios debajo los puentes) se convierten en territorios de negociación y contestación y, a la vez, en espacios donde se construyen sus identidades y se forman comunidades alternativas. Los niños de la calle, estigmatizados por el estado, tratados por los medios de comunicación como delincuentes y como víctimas por las ONG, responden a la marginalización y a la subordinación a través de sus propios cuerpos a partir de vestuario determinado, con tatuajes, piercing, etc.

La escuela resulta un espacio singular para visibilizar las experiencias y prácticas espaciales de los chicos y chicas porque en él no sólo se aprenden temáticas curriculares sino que también se establecen las jerarquías sociales y las relaciones entre iguales. En este espacio, Evans (2006) analiza en Liverpool (Reino Unido) las razones por las cuales las chicas hacen menos deporte que los chicos en la escuela, siendo el miedo a la masculinización del cuerpo y la importancia de la propia imagen algunos factores explicativos.

Finalmente, cabe mencionar dos trabajos que analizan los cuerpos móviles, tema de especial relevancia en un contexto de globalización y movilidad creciente. Los cuerpos en movimiento son estudiados desde distintos enfoques por Hyndman y Alwis (2004) y Gorman-Murray (2009). La primera aportación parte de la consideración de que el cuerpo de las personas es la mejor escala de análisis del espacio político. En una situación de conflicto como el vivido en Sri Lanka el desplazamiento interno de sus habitantes resulta extremadamente difícil y inseguro. Mientras el gobierno fija la identidad de los cuerpos (supuestamente inalterable) a través de los carnets de identidad, las personas visibilizan dimensiones específicas de su identidad (formas de hablar, indumentaria, cómo se 'presenta uno mismo') para circular más fluidamente por carreteras llenas de controles. La segunda contribución, la de Gorman-Murray (2009), permite entender el cuerpo como vector de movimiento, vislumbra como la sexualidad corporal (con sus identidades, sentimientos, deseos y relaciones íntimas) refuerza 
la migración queer y muestra de qué forma las emociones corporales (sentimientos de confort, pertenencia, deseo y amor) juegan un papel primordial en el fortalecimiento del sentido de lugar en el país de destino.

\section{Los cuerpos en la geografía española y latinoamericana}

La geografía feminista y la geografía postmodernista de principios del siglo XXI han impulsado nuevos temas de estudio sobre las sexualidades, los cuerpos y las masculinidades (Garcia Ramon, 2005 y 2008). En España, aunque escasos, han aparecido en los últimos años estudios geográficos sobre los dos primeros. Por un lado, se encuentran los estudios que relacionan el espacio geográfico y la cultura homosexual (Fernández, 2007) y los espacios homosexuales de la ciudad (García Escalona, 2000; Santos, 2002 y 2006) y, por otro, los que incluyen el cuerpo como categoría de análisis y aportan nuevas formas de entender el poder, el conocimiento y las relaciones entre personas y lugares.

Sobre el cuerpo en particular se ha trabajado poco, pudiéndose resaltar como excepción el trabajo de la geógrafa Josepa Bru de la Universitat de Girona publicado en Las Otras Geografias (uno de los principales manuales de geografía de los últimos años, editado Joan Nogué y Joan Romero). Para Bru (2006: 487) el cuerpo representa simultáneamente el primer lugar donde penetra el poder y donde se ofrece resistencia: "[la] geografía del cuerpo como lugar colonizado, traspasado, modelado por el poder pero que, a su vez, a través de un proceso transescalar de autoconciencia, resignificación y reapropiación, contiene el embrión para ofrecerle resistencia”. A partir del análisis de situaciones degradantes como la esclavización, el tráfico de seres humanos, los trabajos forzados, la prostitución, la pornografía y el comercio de la reproducción humana, muestra como el cuerpo se reduce muchas veces a ser mostrado, mirado, manipulado y entendido como instrumento de poder, dominio y seducción. En un trabajo posterior, Bru (2009), y en el marco de la teoría postestructuralista, elabora una excelente reflexión sobre el cuerpo y la palabra como constructoras de la identidad que nos 'sitúan' en el mundo y aboga por "una nueva manera de estar ahí, en el mundo. Un paisaje de seres humanos cuya palabra alienta la insondable belleza de la acogida del otro, de lo otro en mí” (p. 79).

Otros trabajos recientes son los de Rodó-de-Zárate (2011 y 2013). A partir de una revisión bibliográfica, la autora analiza la visibilidad del cuerpo joven sexuado en los espacios públicos urbanos, resaltando como el patriarcado condiciona las prácticas y las experiencias en estos espacios. Dedica un apartado al 'cuerpo' en geografía donde se revisan numerosos artículos dedicados al estudio de éste como lugar de intersección de opresiones y resistencias. 
En América Latina, la Revista Latinoamericana de Estudios sobre cuerpos, emociones y sociedad publicó su primer número en el año 2009 y según la presentación de su web: una iniciativa destinada a brindar un espacio de reflexión, análisis y estudio sobre las temáticas vinculadas a los Cuerpos y las Emociones en y desde América Latina. En el primer número de la revista, la geógrafa y socióloga Alicia Lindón de la Universidad Autónoma Metropolitana de México publica un artículo sobre la geografía y el cuerpo, concibiendo a este último "como una ventana para comprender la construcción social de la ciudad, de lo urbano y sus lugares, a través de los sujetos que la habitan corporal y emocionalmente" (p. 6). A partir de la idea de 'sujeto cuerpo' y 'sujeto sentimiento' esta geógrafa dibuja una serie de escenarios urbanos (móviles y fugaces, fijos e insertos en el ciclo de vida, de la apropiación corporal y efímera) para conocer cómo se experimenta la ciudad desde múltiples corporalidades y estados emocionales. En otro trabajo Lindón (2012), aboga por una mirada transversal de las disciplinas para analizar las interacciones entre el cuerpo, la corporeidad, las emociones, la cotidianidad y los espacios de vida de los sujetos, y comprender mejor la producción y la reproducción socio-territorial.

En la misma revista citada anteriormente, se encuentra el trabajo de los brasileños Alves y Guimaraes (2010) que, desde una perspectiva geográfica, y a partir de entrevistas con mujeres que tuvieron cáncer de mama, estudian las relaciones que las mujeres mantienen con su propio cuerpo una vez mastectomizado, así como con su propia casa, los hospitales donde hicieron el tratamiento, su barrio y su ciudad.

Por último, cabe señalar el artículo publicado por otro geógrafo de la Universidad Autónoma Metropoliatana de México, Cristóbal Mendoza (2012), que se acerca al estudio del cuerpo desde la sexualidad. Sorprende encontrar un tema tan sugerente en medio de tanta escasez productiva. A través de un estudio con trabajadores sexuales en Puerto Vallarta (México), el autor señala que el turista sexual busca la Otredad teniendo en el imaginario los estereotipos de las imágenes occidentales del hombre mexicano (por ejemplo, la piel oscura como representación del macho mexicano).

\section{El cuerpo como herramienta de investigación}

Como seres corpóreos constituidos con múltiples ejes de poder basados en el género, la etnia y la clase social, nuestra identidad como investigadores/as tiene implicaciones importantes para la construcción de las preguntas de investigación y la interpretación de los resultados de la investigación. Sin embargo, muy a menudo, la posicionalidad del investigador/a se describe solamente en relación al género, la edad o la etnia; y otros aspectos relacionados con el cuerpo y el espacio (los olores, los gustos, los gestos, las reacciones, el vestido y las miradas) pasan totalmente desapercibidos en todo el proceso de la investigación. 
"En las prácticas diarias del trabajo de campo fantaseaba con salir de mi cuerpo para crear/sostener la ilusión de la objetividad" (Sundberg, 2005: 20). Las reflexiones de Sundberg (2005) sobre la posicionalidad, la reflexividad, las relaciones de poder y la ética en el trabajo de campo resultan muy interesantes porque muestran hasta qué punto el cuerpo es un ente activo en el proceso de teorización y escritura. Se resalta la importancia de situar nuestro conocimiento y de aceptar que nuestros cuerpos 'hablan' en nuestra forma de escribir; a la vez que se destaca que la investigación se confirma desde nuestras relaciones corporizadas y prácticas de negociación y, por tanto, es siempre selectiva y parcial.

Las técnicas cualitativas más frecuentes en el estudio de las geografías de los cuerpos, los lugares y las emociones son las entrevistas semi-estructuradas, los grupos de discusión, la observación participante y los diarios. Sin embargo, pocos trabajos analizan el papel del cuerpo como herramienta de investigación. Lo hacen Bain y Nast (2006) y Longhurst et al. (2008) a partir de la premisa de que los cuerpos están siempre interpelados por un abanico de prácticas ideológicas, inclusive cuando están en el 'campo', y de la idea de que quien investiga y participa tiene subjetividades corporales distintas.

Tres investigaciones resultan especialmente innovadoras en sus propuestas metodológicas. En primer lugar, encontramos el trabajo de Bain y Nast (2006) en el cual describen detalladamente algunas secuencias del proceso de la investigación (cómo se visten para asistir a una sauna queer, qué movimientos hacen una vez dentro y con quien interactúan) y explican muy abiertamente sus experiencias como insiders y outsiders, participantes y a la vez investigadoras. En segundo lugar, y como un intento de generar datos cualitativos distintos, Longhurst et al. (2008) exploran la vida de mujeres migrantes (refugiadas, estudiantes internacionales y trabajadoras temporales) a través de la comida. Para ello reúnen en una comida a un grupo de mujeres migrantes y observan las reacciones de sus cuerpos, con sus olores, gestos, tonos de voz y comportamientos. Así, pues, para las autoras el reconocimiento de todos nuestros sentidos (táctil, olfativo, gusto, auditivo y visual) tiene la potencialidad de enriquecer el conocimiento de las relaciones entre cuerpo y espacio. Finalmente, Brown (2008) participa, ya no como observador o manteniendo conversaciones informales, sino como actor activo (y corporal) en los encuentros homoeróticos en lugares públicos, documentándolos con un diario de campo, con mapas de los lugares y diagramas coreográficos de los encuentros (según la terminología empleada por el mismo autor). En el marco de la teoría no-representacional (en la que los cuerpos son entendidos por su materialidad, con sus gestos, movimientos y performatividades mismas), el geógrafo considera el sexo como práctica espacial y examina las microgeografías del encuentro en los espacios públicos urbanos. 


\section{Reflexiones finales}

Tradicionalmente la geografía no había considerado que el cuerpo fuese un lugar, que ocupase un espacio o que a través de él experimentásemos nuestras emociones. Sin embargo, cada vez más y, concretamente, la geografía feminista anglosajona, ha ido reconociendo todas estas características, así como el carácter fluido de la identidad de los sujetos y la identidad del cuerpo (con su género, sus preferencias sexuales, sus habilidades físicas, su edad, su color y su etnicidad). En la geografía cultural española (así como en la latinoamericana) la consideración del cuerpo como escala de análisis y herramienta de investigación es aún incipiente comparada con la del mundo anglosajón. En este contexto geográfico (Reino Unido, Estados Unidos, Australia y Nueva Zelanda, especialmente), las más prestigiosas revistas de geografía han publicado a inicios del siglo XXI artículos relacionados con la fluidez de los cuerpos, la construcción de la identidad a través de ellos, los cuidados corporales interpersonales, los cuerpos discapacitados y enfermos, entre otros intereses de estudio. Conocer estos trabajos y ver en qué punto nos encontramos puede ser de gran ayuda a la hora de mostrar otras formas de 'hacer' y 'entender' la geografía, así como, a la hora de avanzar en la conceptualización de la geografía cultural española.

\section{Agradecimientos}

Agradezco muy sinceramente la lectura y comentarios que han hecho de este artículo Maria Dolors Garcia Ramon, Maria Prats Ferret y Maria Rodó-de-Zárate de la Universitat Autònoma de Barcelona y Paloma Puente Lozano de la Universidad Carlos III de Madrid; así como la evaluación y los comentarios críticos de dos evaluadores/as de la revista.

Una primera versión de este artículo fue presentado en forma de ponencia al Simposio: "Cuerpo, experiencia y textualidad: una aproximación metodológica", en el Critical Social Psychology Congress: discourse, materiality and polítics, celebrado en la Universitat Autònoma de Barcelona, del 6 al 8 de febrero de 2013.

\section{Bibliografía}

Abrahamsson, S. y Simpson, P. (2011) The limits of the body: boundaries, capacities, thresholds. Social and Cultural Geography, 12 (4), 331-338.
Alves, N.C. y Guimaraes, R.B. (2010) Escala geográfica, cáncer do mama e corpo feminino. Revista Latino-Americana de Geografía e Gênero, 1 (2), 245-253. 
Ansell, N. (2009) Embodied learning: responding to AIDS in Lesotho's education sector. Children's Geographies, 7(1), 21-36.

Atkinson, S.; Lawson, V. y Wiles, J. (2011) Care of the body: spaces of practice. Social and Cultural Geography, 12(6), 563-572.

Atkinson, S. (2011) Scales of care and responsability: debating the surgically globalised body. Social and Cultural Geography, 12(6), 623-637.

Bain, A.L. y Nash, C. (2006) Undressing the researcher: feminism, embodiment and sexuality at a queer bathouse event. Area, 38(1), 99-106.

Beazley, H. (2003) Voices from the margins: street children's subcultures in Indonesia. Children's Geographies, 1(2), 181-200.

Bru, J. (2006) El cuerpo como mercancía. En Nogué, J. y Romero, J. (eds.) Las Otras Geografias, 465-491, València, Tirant lo Blanch.

Bru, J. (2009) Cuerpo y palabra o los paisajes de la cautividad. En Nogué, J. (ed.) La construcción social del paisaje, Madrid, Biblioteca Nueva.

Brown, G. (2008) Ceramics, clothing and other bodies: affective geographies of homoerotic cruising encounters. Social and Cultural Geography, 9(8), 915-932.

Butler, R. (1999) The body. En Cloke, P., Crang, P. y Goodwin, M. (eds.) Introducing human geographies, London, Arnold.

Colls, R. (2004): 'Looking alright, feeling alright': emotions, sizing and the geographies of women's experiences of clothing consumption. Social and Cultural Geography, 5(4), 583-596.

Colls, R. (2006) Outsize/Outside: Bodily bignesses and the emotional experiences of British women shopping for clothes. Gender, Place \& Culture, 13(5), 529-545.
Colls, R. (2007) Materialising bodily matter: intra-action and the embodiment of 'Fat'. Geoforum, 38, 353-365.

Colls, R. y Hörschelmann, K. (2009) The geographies of children's and Young people's bodies. Children's Geographies, 7(1), 1-6.

Crewe, L. (2001) The besieged body: geographies of retailing and consumption. Progress in Human Geography, 25(4), 629-640.

Davidson, J.; Bondi, L. y Smith, M. (2007) Emotional Geographies. Aldershot, Ashgate.

Dyer, S.; McDowell, L. y Batnitzky, A. (2008) Emotional labour/body work: the caring labours of migrants in the UK's National Health Service. Geoforum, 39, 2030-2038.

Evans, B. (2006) I'd Feel Ashamed': Girls' Bodies and Sports Participation. Gender, Place E Culture, 13(5), 547-561.

Fernández Salinas, V. (2007) Comunidad gay y espacio en España. Boletín de la Asociación de Geógrafos Españoles, 43, 241-260.

Garcia Escalona, E. (2000) "Del armario al barrio": aproximación a un nuevo espacio urbano. Anales de Geografía de la Universidad Complutense, 20, 437-449.

Garcia Ramón, M.D.; Albet, A. y Zusman, P. (2003) Recent developments in social and cultural geography in Spain". Social and Cultural Geography, 4 (3), 419-431.

Garcia Ramón, M.D. (2005) Respondiendo a un desafío pendiente en geografía: el enfoque de género visto desde España. Geographicalia, 48, 55-75.

Garcia Ramón, M.D. (2008) ¿Espacios sexuados o masculinidades y feminidades espaciales?: hacia una geografía del género. $S E-$ MATA. Ciencias Sociais e Humanidades, 20, 25-51.

Gökariksel, B. (2009) Beyond the officially sacred: religión, secularism and the body in the production of subjectivity. Social and Cultural Geography, 10(6), 657-674. 
Gorman-Murray, A. (2009) Intimate mobilities: emotional embodiment and queer migration. Social and Cultural Geography, 10(4), 441-460.

Herrera, E., Jones, G.A. y Thomas de Benitez, S. (2009) Bodies on the line: identity markers among Mexican Street youth. Children's Geographies, 7(1), 67-81.

Herrick, C. (2007) Risky bodies: public health, social marketing and the governance of obesity. Geoforum, 38, 90-102.

Hopkins, P.E. (2010) Young people, place and identity. London, Routledge.

Hyndman, J. y Alwis, M. de (2004) Bodies, Shrines, and Roads: violence, (im)mobility and displacement in Sri Lanka. Gender, Place \& Culture, 11(4), 535-557.

Johnston, L. (1996) Flexing Femininity: Female body-builders refiguring 'the body'. Gender, Place \& Culture, 3(3), 327-340.

Landzelius, M. (2005). The body, En Duncan, J.S., Johnson N.C. y Schein, R.H. (eds.) $A$ companion to cultural geography, 279-297, Oxford, Blackwell.

Lea, J. (2009) Becoming skilled: the cultural and corporeal geographies of teaching and learning Thai Yoga massage. Geoforum, 40, 465-474.

Lindon, A. (2009) La construcción socioespacial de la ciudad: el sujeto cuerpo y el sujeto sentimiento. Cuerpos, emociones y sociedad, 1, 6-20.

Lindon, A. (2012) Corporalidades, emociones y espacialidades. Hacia un renovado betweenness. Revista Brasileira de Sociologia da Emoçao, 1(33), 698-722.

Little, J. (2013) Pampering, well-being and women's bodies in the therapeutic spaces of the spa. Social and Cultural Geography, 14(1), 41-58.
Longhurst, R. (1997) (Dis)embodied geographies. Progress in Human Geography, 21(4), 486-501.

Longhurst, R. (1999) Pregnant bodies, public scrutiny. 'Giving' advice to pregnant women. En Teather, E.K. (ed.) Embodied geographies. Spaces, bodies and rites of passage, London, Routledge.

Longhurst, R. (2001) Bodies: exploring fluid boundaries, London, Routledge.

Longhurst, R. (2005) (Ad)dressing pregnant bodies in New Zealand: clothing, fashion, subjectivities and spatialities. Gender, Place and Culture, 12(4), 433-446.

Longhurts, R., Ho, E. y Johnston, L. (2008) Using 'the body' as an 'instrumental of research': kimch'i and pavlova. Area, 40(2), 208-217.

Malins, P., Fitzgerald, J.L. y Threadgold, T. (2006) Spatial 'folds': the entwining of bodies, risks and city spaces for women injecting drug users in Melbourne's Central Business District. Gender, Place and Culture, 13(5), 509-527.

Mather, C. y Mackenzie, C. (2006) The body in transnational commodity cultures: South Africa's Outspan 'girls' campaign. Social and Cultural Geography, 7(3), 403-420.

McCormack, D. (1999) Body shopping: reconfiguring geographies of fitness. Gender, Place and Culture, 6(2), 155-177.

Mendoza, C. (2012) Beyond sex tourism: gay tourism and male sex workers in Puerto Vallarta (Western Mexico). International Journal of Tourism Research, 15(2), 122-137.

Moss, P. y Dyck, I. (1996) Inquiry into environment and body: women, work and chronic illness. Environment and Planning D: Society and Space, 14, 737-753.

Moss, P. y Dyck, I. (1999) Journeying through M.E. Identity, the body and women with chronic illness. En Teather, E.K. (ed.) Em- 
bodied geographies. Spaces, bodies and rites of passage, London, Routledge.

Parr, H. (2002a) Medical geography: diagnosing the body in medical and health geography, 1999-2000. Progress in Human Geography, 26(2), 240-251.

Parr, H. (2002b) New body-geographies: the embodied spaces of health and medical information on the Internet. Environment and Planning D: Society and Space, 20, 73-95.

Prats, M., Baylina, M. y Ortiz, A. (2012) Los lugares de la amistad y la vida cotidiana de chicas y chicos adolescentes en un barrio de Barcelona. Revista Latino-Americana de Geografia e Gênero, 3(2).

$<$ http://www.revistas2.uepg.br/index.php /rlagg/article/viewArticle/3355>

(consulta: 17.01. 2013).

Rodó-de-Zárate, M. (2011) El jovent i els espais públics urbans des de la perspectiva de gènere: Un estat de la qüestió des de la geografia. Documents d'Anàlisi Geogräfica, 57(1), 147-162.
Rodó-de-Zárate, M. (2013) Gènere, cos i sexualitat. La joventut, l'experiència i l'ús de l'espai públic urbà. Papers, 98(1), 127-142.

Santos, X.M. (2002) Espacios disidentes en los procesos de ordenación territorial. Documents d'Anàlisi Geogrâfica, 40, 69-104.

Santos, X.M. (2006) Espacios disidentes homosexuales. Nogué, J. y Romero, J. (eds.) Las otras Geografías, 465-491, València, Tirant lo Blanch.

Sothern, M. (2007) You could truly be yourself if you just weren't you: sexuality, disabled body space, and the (neo)liberal politics of self-help. Environment and Planning D: Society and Space, 25, 144-159.

Sundberg, J. (2005) Looking for the critical geographer, or why bodies and geographies matter to the emergence of critical geographies of Latin America. Geoforum, 36, 17-28.

Wainwright, E., Buckingham, S., Marandet, E. y Smith, F. (2010) 'Body training': investigating the embodied training choices of/for mothers in West London. Geoforum, 41, 489-497. 
